\title{
The Chiral Transition in QCD: on the quark mass dependence of Goldstone fluctuations
}

\author{
Wolfgang Unger* for the RBC-Bielefeld Collaboration \\ University Bielefeld \\ E-mail: wunger@physik.uni-bielefeld.de
}

We analyze the quark mass dependence of the chiral condensate and chiral susceptibilities in QCD with two degenerate light quark masses and a strange quark mass close to its physical value. We provide evidence for the influence of thermal fluctuations of Goldstone modes on the chiral condensate at finite temperature. We show that at temperatures below but close to the chiral phase transition at vanishing quark mass this leads to a characteristic dependence of the light quark chiral condensate on the square root of the light quark mass $\left(m_{l}\right)$. As a consequence the chiral susceptibility shows a strong quark mass dependence for all temperatures below $T_{c}$ and diverges like $1 / \sqrt{m_{l}}$ the in chiral limit. We separately examine the divergence of disconnected and connected parts of the light quark susceptibility and discuss the cut-off dependence of susceptibilities and chiral condensates.

The XXVII International Symposium on Lattice Field Theory

July 26-31, 2009

Peking University, Beijing, China

${ }^{*}$ Speaker. 


\section{Introduction}

Establishing the properties of the chiral phase transition in QCD is an important problem in lattice simulations of QCD thermodynamics. Although several attempts have been undertaken to establish the universal scaling behavior in the limit of vanishing light quark masses no clear-cut evidence for the expected $O(N)$ scaling, related to the underlying chiral symmetry of the QCD Lagrangian, has been found. In this paper we will discuss an effect that influences the scaling behavior of thermodynamic quantities, in particular chiral susceptibilities, in the chirally broken phase. The physics of QCD at low energies is expected to be described by $\mathrm{O}(\mathrm{N})$ symmetric spin models due to the group theoretic isomorphism ${ }^{1} \mathrm{SU}(2)_{L} \times \mathrm{SU}(2)_{R} \simeq \mathrm{O}(4) \rightarrow \mathrm{O}(3) \simeq \mathrm{SU}(2)_{V}$. The $\mathrm{O}$ (4) fields correspond to the meson fields $\pi^{i} \sim \bar{\psi} \gamma_{5} t^{i} \psi$ and $\sigma \sim-\bar{\psi} \psi$, the explicit symmetry breaking external field $H$ is proportional to the quark mass $m_{l}$ and the order parameter is $\Sigma=\langle\sigma\rangle$. This description is widely assumed to be valid below and in the vicinity of the transition temperature.

\section{The Goldstone Effect}

$\mathrm{O}(\mathrm{N})$ spin models in 3 and 4 dimensions have been studied extensively [1, 2]. It was shown within chiral perturbation theory that $N-1$ transverse Goldstone modes give corrections to $\Sigma$ in case of a non-zero external field $H$. In three dimensions, which may be applicable to QCD close to the critical temperature, one finds:

$$
\Sigma_{H}=\Sigma_{0}\left(1+\frac{N-1}{8 \pi} \frac{\left(\Sigma_{0} H\right)^{1 / 2}}{F_{0}^{3}}+\mathscr{O}(H)\right)
$$

Besides Goldstone scaling below $T_{c}$, one expects critical behavior at $T_{c}$ :

$$
\Sigma(h=0, t<0) \sim|t|^{\beta}, \quad \text { and } \quad \Sigma(h, t=0) \sim h^{1 / \delta}, \quad t=\frac{1}{t_{0}} \frac{T-T_{c}}{T_{c}}, \quad h=H / h_{0}
$$

The Goldstone scaling, Eq. (2.1), is encoded in the $\mathrm{O}(\mathrm{N})$ scaling function $f_{G}(z)$ (with $z=t / h^{1 / \beta \delta}$ ), which can be seen by considering the magnetic equation of state [3]:

$$
\Sigma(h, t)=h^{1 / \delta} f_{G}(z), \quad f_{G}(z) \simeq(-z)^{\beta}\left(1+\tilde{c}_{2} \beta(-z)^{-\beta \delta / 2}\right) \quad \text { for } \quad z \rightarrow-\infty
$$

The corresponding susceptibility, defined by $\chi \equiv \frac{\partial \Sigma}{\partial H}$ then scales as $\chi \sim h^{-1 / 2}$ for $t<0$ and as $\chi \sim h^{1 / \delta-1}$ for $t=0$. This behaviour is encoded in a scaling function $f_{\chi}(z)$ which is related to $f_{G}(z)$. The $\mathrm{O}(2)$ and $\mathrm{O}(4)$ scaling functions were studied numerically [4]. We have shown recently that also our lattice QCD data for $N_{\mathrm{f}}=2+1$, on which also this paper is based on, are well described by the scaling function $f_{G}$ for small quark masses [5].

The leading order quark mass dependence in $N_{\mathrm{f}}=2+1 \mathrm{QCD}$ is on the same footing as the $H$ dependence in $\mathrm{O}(2) / \mathrm{O}(4)$ models: both results are obtained via chiral perturbation theory. The effective Lagrangian is to lowest order the one of the nonlinear sigma model and contains two low energy constants, $F_{0} \approx 93 \mathrm{MeV}$ being the Pion decay constant in the chiral limit, and $B_{0}$ which

\footnotetext{
${ }^{1} \mathrm{O}(4)$ critical behaviour is still expected for $N_{\mathrm{f}}=2+1$, as long as the strange quark mass is sufficiently large, namely above the tricritical point $m_{s}^{\text {tric. }}$. The location of the tricritical point is however not known yet.
} 
parametrizes the condensate, $B_{0}=-\lim _{m_{l} \rightarrow 0}\langle 0|\bar{\psi} \psi| 0\rangle / N_{1} F_{0}^{2}$, as well as the Gell-Mann, Oakes, Renner relation $M_{0}=\lim _{m_{l} \rightarrow 0}\left(2 m_{l} B_{0}\right)^{1 / 2}$. At finite temperature [1,6], one obtains an expression for the chiral condensate, valid close to the chiral limit:

$$
\langle\bar{\psi} \psi\rangle=\langle 0|\bar{\psi} \psi| 0\rangle\left\{1+\frac{N_{1}^{2}-1}{N_{1}}\left[-\frac{\left(T / F_{0}\right)^{2}}{12}+\frac{1}{4 \pi} \frac{T M_{0}}{F_{0}^{2}}+\frac{1}{8 \pi^{2}}\left(\frac{M_{0}}{F_{0}}\right)^{2} \log \left(\frac{M_{0}}{4 \pi T}\right)+\mathscr{O}\left(M_{0}{ }^{3}\right)\right]\right\}
$$

In this expression the Goldstone term is to be identified with the term linear in $M_{0}$. From the condensate one can easily obtain the full chiral susceptibility [7]. Decomposed into connected and disconnected part $^{2}$ one obtains for the infrared part, which stems from the Goldstone modes, for $N_{1}$ degenerate light flavors:

$$
\chi_{\mathrm{con}}^{\mathrm{IR}}=\frac{N_{1}^{2}-4}{8 \pi} \frac{T}{\sqrt{2 m_{l}}}\left(\frac{\Sigma}{F_{0}^{2}}\right)^{3 / 2} \quad \chi_{\mathrm{dis}}^{\mathrm{IR}}=\frac{N_{1}^{2}+2}{8 \pi} \frac{T}{\sqrt{2 m_{l}}}\left(\frac{\Sigma}{F_{0}^{2}}\right)^{3 / 2}
$$

Note that in the continuum no Goldstone divergence is expected in the connected part for $N_{1}=2$.

\section{Susceptibilities from Staggered Chiral Perturbation Theory}

The lattice discretization scheme for staggered fermions leads to lattice artifacts known as taste breaking. As a result, the flavor symmetries are only approximate, which also results in a redcued symmetry of the Lagrangian: the lattice breaks chiral symmetry down to axial $\mathrm{U}(1)$, hence one expects $\mathrm{O}(2)$ instead of $\mathrm{O}(4)$ critical scaling in the chiral limit. To reduce taste breaking we make use of an improved lattice action, the p4fat 3 action [10]. Taste breaking also affects the pseudoscalar meson masses, which for nonzero lattice spacing will remain nonzero even in the chiral limit. In order to estimate the effect of taste violations we make use of staggered chiral perturbation theory ( $\chi$ PT). This is based on the Lee-Sharpe Lagrangian which in addition to the nonlinear sigma term contains taste breaking operators. From these, shifts $\Delta_{t}$ of the tree-level masses of pseudoscalar mesons in the taste channel $t$ can be derived:

$$
m_{f, f^{\prime}, t}^{2}=B_{0}\left(m_{f}+m_{f^{\prime}}\right)+a^{2} \Delta_{t}
$$

Here $f, f^{\prime}$ denote the flavors $u, d, s$. The shifts are nonvanishing for all taste channels except for the pseudoscalar one because the taste non-singlet $U(1)$ symmetry is unbroken by the lattice. Hence, $\pi_{P}$ is the only true Goldstone boson.

The effect of taste violations on the Goldstone effect in the chiral susceptibilites can be calculated via integrated correlations functions (so-called bubble terms) for the scalar mesons, $B_{a_{0}}(p), B_{f_{0}}(p)$. The relation between IR-divergent contributions of the bubble terms and chiral susceptibility is:

$$
\chi_{\mathrm{con}}^{\mathrm{IR}}=B_{a_{0}}^{\mathrm{IR}}(p=0), \quad \chi_{\mathrm{dis}}^{\mathrm{IR}}=B_{f_{0}}^{\mathrm{IR}}(p=0)-B_{a_{0}}^{\mathrm{IR}}(p=0)
$$

\footnotetext{
${ }^{2}$ The connected susceptibility [8] and the full susceptiblity can be calculated within chiral perturbation theory, the disconnected susceptibility is then obtained by subtracting the connected susceptibility from the full susceptibility [9].
} 
Computation of the bubble terms via s $\chi \mathrm{PT}$ yields at zero momentum $[11,12,13]:{ }^{3}$

$$
\begin{aligned}
& B_{a_{0}}(0) \sim \sum_{k}\left[\sum_{f, t} \frac{n_{\mathrm{r}}^{2}}{\left(k^{2}+m_{f u, t}^{2}\right)^{2}}-\frac{2}{\left(k^{2}+m_{\pi, I}^{2}\right)^{2}}+\frac{2 / 3}{\left(k^{2}+m_{\pi, I}^{2}\right)\left(k^{2}+m_{\eta, I}^{2}\right)}+\mathscr{O}^{V / A}\right] \\
& B_{f_{0}}(0)-B_{a_{0}}(0) \sim \sum_{k}\left[\sum_{t} \frac{2 n_{\mathrm{r}}^{2}}{\left(k^{2}+m_{\pi, t}^{2}\right)^{2}}+\frac{4}{9}\left(\frac{1 / 2}{k^{2}+m_{\pi, I}^{2}}+\frac{1}{k^{2}+m_{\eta, I}^{2}}\right)^{2}+\mathscr{O}^{V / A}\right]
\end{aligned}
$$

In the continuum limit the sum of so-called hairpin propagators in the V/A taste channels $\mathscr{O}^{V / A}$ as well as the taste violations in the meson masses vanish $\sim a^{2}$ and we obtain the continuum result:

$$
\begin{aligned}
& B_{a_{0}}(0) \sim \sum_{k}\left[\frac{32 n_{\mathrm{r}}^{2}}{\left(k^{2}+m_{\pi}^{2}\right)^{2}}+\frac{16 n_{\mathrm{r}}^{2}}{\left(k^{2}+m_{K}^{2}\right)^{2}}-\frac{2}{\left(k^{2}+m_{\pi}^{2}\right)^{2}}+\frac{2 / 3}{\left(k^{2}+m_{\pi}^{2}\right)\left(k^{2}+m_{\eta}^{2}\right)}\right] \\
& B_{f_{0}}(0)-B_{a_{0}}(0) \sim \sum_{k}\left[\frac{32 n_{\mathrm{r}}^{2}}{\left(k^{2}+m_{\pi}^{2}\right)^{2}}+\frac{1}{\left(k^{2}+m_{\pi}^{2}\right)^{2}}+\frac{1 / 9}{\left(k^{2}+m_{\eta}^{2}\right)^{2}}-\frac{2 / 3}{\left(k^{2}+m_{\pi}^{2}\right)\left(k^{2}+m_{\eta}^{2}\right)}\right]
\end{aligned}
$$

By setting $n_{\mathrm{r}}=1 / 4$ one can clearly see that for $B_{a_{0}}$ the $\pi \pi$ states cancel, hence there is no Goldstone mode in the continuum, whereas for $B_{f_{0}}$ we end up with three $\pi \pi$ states, as expected. However, with large taste violations one obtains Goldstone modes in $B_{a_{0}}$ and $B_{f_{0}}$ only in the pseudo-taste channel, where $\Delta_{P}=0$ :

$$
B_{a_{0}}^{I R}(0) \sim \sum_{k} \frac{2 / 16}{\left(k^{2}+m_{\pi, P}^{2}\right)^{2}}, \quad B_{f_{0}}^{I R}(0) \sim \sum_{k} \frac{4 / 16}{\left(k^{2}+m_{\pi, P}^{2}\right)^{2}}
$$

As a consequence, by comparing Eq. (3.7) with Eqs. (3.5), (3.6) via the identity (3.2), one expects to find the Goldstone prefactor for the disconnected part to be 24 times larger in the continuum than on a coarse lattice, and the one for the full susceptiblity 12 times larger. Hence, the prefactor for the condensate is also 12 times larger in the continuum than on a coarse lattice.

\section{Preliminary Results on Goldstone Scaling}

This analysis is based on configurations with fixed lattice spacing, $N_{\tau}=4$, generated by the RBCBielefeld Collaboration. Independent of the size of the lattice artifacts, Goldstone modes should be present. In our simulation we used the Symanzik tree-level improved gauge action and the p4fat3 fermion action. The strange quark mass was fixed to $m_{s} a=0.065$, which corresponds to approximately $M_{\bar{s} s}=669 \mathrm{MeV}$. We varied the quark mass between $m_{l}=1 / 5$ to $m_{l}=1 / 80$, hence a mass ratio of $m_{l} / m_{s}=1 / 20$ corresponds to $M_{\pi} \simeq 150 \mathrm{MeV}$ and $m_{l} / m_{s}=1 / 80$ corresponds to $M_{\pi} \simeq 75 \mathrm{MeV}$. For simulation details and tables on statistics and expectation values see [5].

\footnotetext{
${ }^{3}$ Here, $n_{r}$ is the replica dimension: Rooting i.e taking the fourth root of the fermion determinant amounts to setting $n_{\mathrm{r}}=1 / 4$. The quark-line connected propagators are a straightforward modification of the continuum scalar propagator. However, there are also quark-line disconnected propagators in the $t=V, A$ and $I$ taste channel, due to double trace terms in the effective potential. They give rise to so-called hairpin diagrams, as a result the masses of the $\eta$ and $\eta^{\prime}$ in these taste channels are more involved. We will neglect these subtleties because it will not be relevant to the final result. Also, we consinder the limit of a strong axial anomaly, $m_{0} \rightarrow \infty$, and hence neglect the contribution of the $\pi-\eta^{\prime}$ channel.
} 
At non-vanishing quark mass $m_{l}$, the chiral condensate on the lattice receives a contribution which is quadratically divergent in the UV and which in the following ansatz is parametrized by the coefficient $c_{\mathrm{UV}}$ :

$$
\langle\bar{\psi} \psi\rangle_{l}(T)=\langle\bar{\psi} \psi\rangle_{m_{l}=0}(T)+c_{1}(T) \sum_{t=1}^{16} \sqrt{2 B_{0} m_{l}+\Delta_{t} a^{2}}+c_{2}(T) m_{l}+c_{U V} m_{l} / a^{2}+\mathscr{O}\left(m_{l}^{3}\right)
$$

In addition to $\langle\bar{\psi} \psi\rangle_{l}$ we also introduce a subtracted order parameter where this additively divergent piece is subtracted: ${ }^{4}$

$$
\Delta_{l, s} \equiv\langle\bar{\psi} \psi\rangle_{l}-\frac{m_{l}}{m_{s}}\langle\bar{\psi} \psi\rangle_{s}=\frac{N_{1}}{4}\left(\left\langle\operatorname{Tr} M_{l}^{-1}\right\rangle-\frac{m_{l}}{m_{s}}\left\langle\operatorname{Tr} M_{s}^{-1}\right\rangle\right)
$$

Goldstone Fits for the Chiral Condensate: For the fits to the chiral condensate data we use a slightly simplified ansatz focussing on the Goldstone contributions, i.e. we approximate $\langle\bar{\psi} \psi\rangle_{q}=a+\bar{b} \sqrt{m_{q}}+\bar{c} m_{q}$ for $q=l, s$. This approximation neglects a light quark mass dependence of $\langle\bar{\psi} \psi\rangle_{s}$ which is weak however. At fixed $m_{s}$ it is more convenient to use $H \equiv m_{l} / m_{s}$ as fit variable, which leads to the fit ansätze:

$$
\langle\bar{\psi} \psi\rangle_{l}(H)=a+b \sqrt{H}+c H, \quad \Delta_{l, s}(H)=a+b \sqrt{H}+(c-a-b) H .
$$

The coefficient $c$ in $\Delta_{l, s}$ accounts for the flavor dependence of $\bar{c}$. We expect it to be small since the leading, flavor independent UV contribution $c_{\mathrm{UV}}$ should drop out. Indeed, we find that $c$ in $\Delta_{l, s}$ is almost zero (see Fig. 1). The fit coefficient $a$ gives the chiral condensate in the chiral limit and the combination of the fit coefficients $b / a^{3 / 2} \sim 1 / F_{0}^{3}$ seems to diverge at $\beta_{c}$, due to $F_{0} \sim|t|^{v / 2}$.
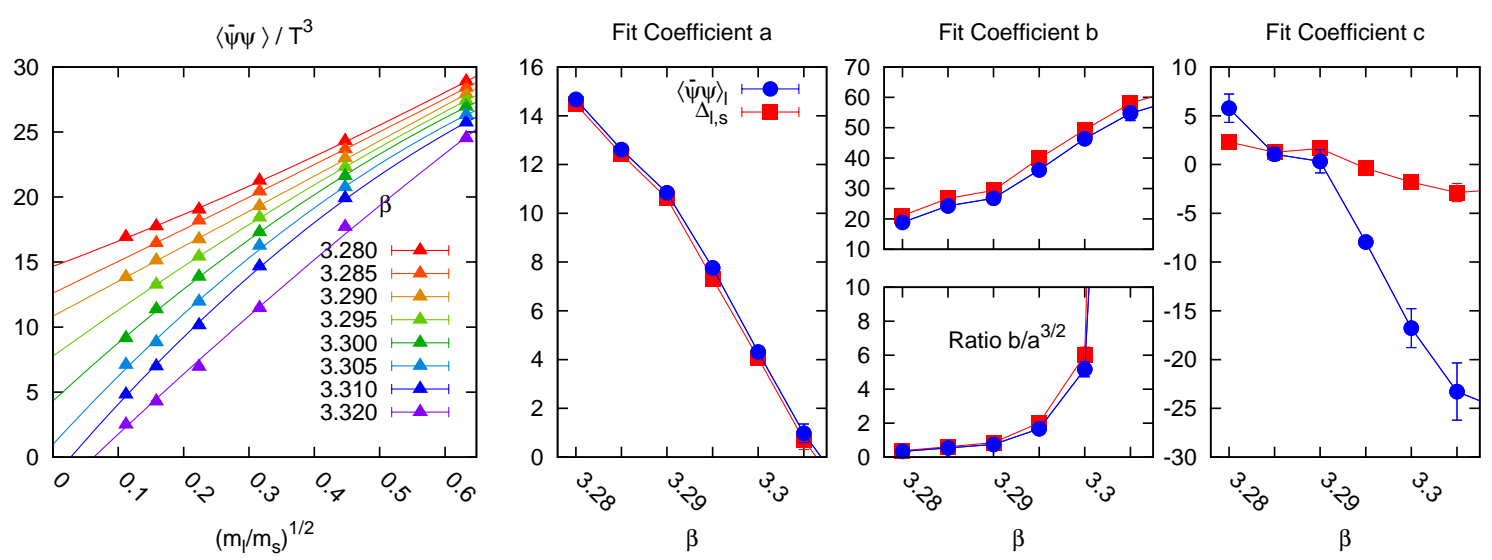

Figure 1: left: Goldstone fit of chiral condensate $\langle\bar{\psi} \psi\rangle$, right: fit coefficients $a, b$ and $c$.

Goldstone Fits for the Chiral Susceptibilities: Being (more) singular in the UV as well as the IR, the connected and disconnected chiral susceptibilities provide additional information about scaling.

\footnotetext{
${ }^{4}$ In all plots we show the 2-flavor condensates and susceptibilites, this implies that one has to subtract twice the 1 -flavor strange-quark susceptilibity in order to remove the leading UV divergent term in $\Delta_{l, s}$. Note that $\Delta_{l, s}$ is not multiplicatively renormalized.
} 
From the $c_{\mathrm{UV}} m_{l}$ term in Eq. (4.1) the full susceptibility inherits a UV divergence as a leading contribution. We have numerical evidence that this is sitting in the connected part, $\chi_{\text {con }}$, only. We estimate this contribution by fitting $\chi_{\text {con }}$ to $c_{\mathrm{UV}}+H^{-1 / 2}$ in the large $H$ limit (see Fig. 3) and subtract this estimate from $\chi_{\mathrm{con}}$ in the following. According to Eq. (3.7) we expect that IR singularities $\sim H^{-1 / 2}$ appear in both, $\chi_{\text {dis }}$ as well as $\chi_{\text {con }}$. We therefore plot the rescaled susceptibilities, $\chi H^{1 / 2}$, in Fig. 2. Indeed, for $m_{l} / m_{s} \leq 1 / 10$ we observe approximate scaling in both quantities for $\beta \leq$ $\beta_{c}=3.300(1)$ which has been identified as the Goldstone regime in [5]. The IR scaling of $\chi_{\text {con }}$ thus signals the presence of taste violations.
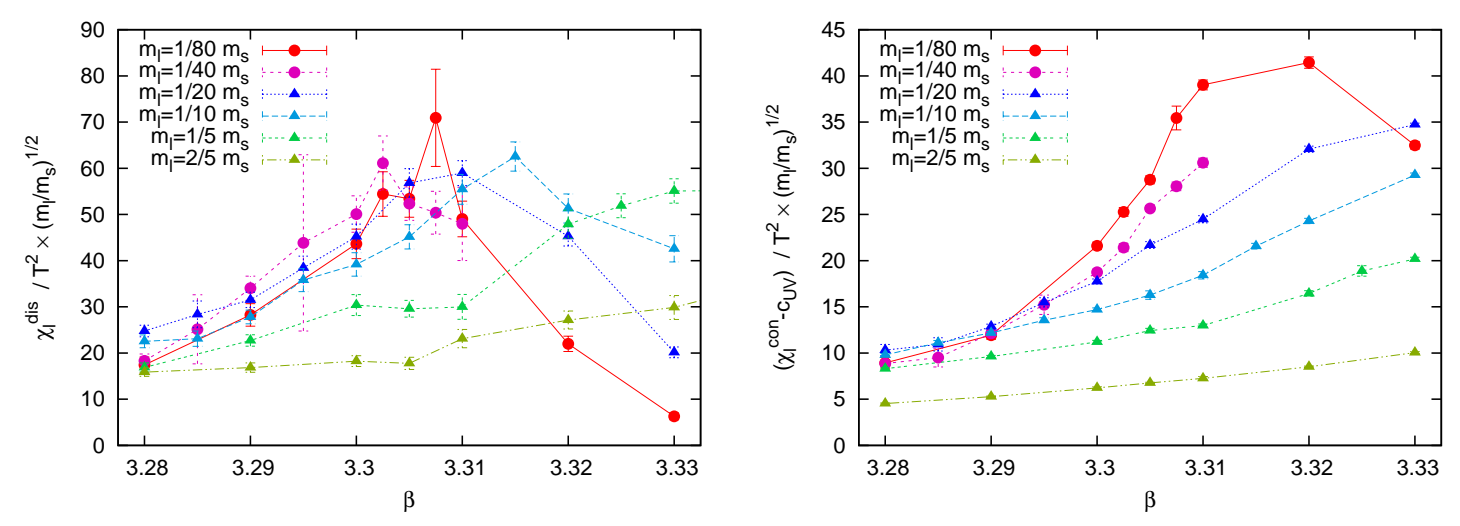

Figure 2: rescaled chiral susceptibilities - left: disconnected part, right: connected part with subtracted UV-divergent constant - in the Goldstone regime, below $\beta_{c}=3.300(1)$, the susceptibilites scale with $H^{1 / 2}$

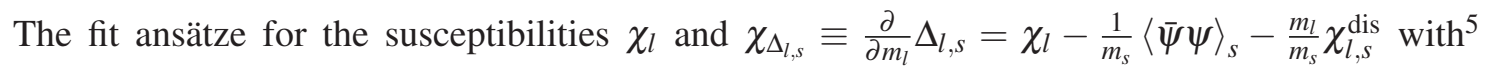
$\chi_{l, s}^{\mathrm{dis}}=\left\langle\operatorname{Tr} M_{l}^{-1} \operatorname{Tr} M_{s}^{-1}\right\rangle-\left\langle\operatorname{Tr} M_{l}^{-1}\right\rangle\left\langle\operatorname{Tr} M_{s}^{-1}\right\rangle$ were derived from the fit ansätze of the condensates by derivation wrt. $m_{l}$ : The fit coefficients of the condensate fits in Fig. 1 and of the susceptibility fits in Fig. 3 can be directly compared with each other. Note that in the fit ansatz for $\chi_{l}$ coefficient $a$ drops out and for $\chi_{\Delta_{l, s}}$ coefficient $c$ drops out. One can see how the coefficients of the full susceptibility, which should agree with those of the condensate, are composed of the sum of the coefficients of its disconnected and connected part. We find qualitatively similar behaviour when comparing the fit coefficients of the full susceptibilties $\left(\chi_{l}\right.$ and $\left.\chi_{\Delta_{l, s}}\right)$ with the coefficients of the condensates. Moreover, the prefactor $b$ for the disconnected part is more than twice as large as for the connected part. From this we can infer that the disconnected part also sees contributions from other taste channels than $t=P$, indicating that taste violations are not large in all taste channels in the quark mass window considered. ${ }^{6}$

\section{Conclusion and Outlook}

The Goldstone effect, as predicted via chiral perturbation theory, predicts an IR divergence for the chiral susceptibility: $\chi \sim 1 / \sqrt{m_{l}}$. Goldstone scaling is consistent with $\mathrm{O}(\mathrm{N})$ scaling which

\footnotetext{
${ }^{5}$ We idendified connected and disconnected terms for the susceptibility of the subtracted order parameter in the following way: $\chi_{\Delta_{l, s}}^{\mathrm{dis}}=\chi_{l}^{\mathrm{dis}}-\frac{m_{l}}{m_{s}} \chi_{l, s}^{\mathrm{dis}}$ and $\chi_{\Delta_{l, s}}^{\text {con }}=\chi_{l}^{\text {con }}-\frac{1}{m_{s}}\langle\bar{\psi} \psi\rangle_{s}$

${ }^{6}$ The fit coefficient $a$, which does not drop out in the fit ansatz for $\chi_{\Delta_{l, s}}$, is smaller as compared to the condensate fit, however, connected and disconnected part seem to have opposite sign. The recovery of $a$ is due to the the strange condensate contribution to $\chi_{\Delta_{l, s}}$ which however vanishes in the chiral limit. This induces systematic error on $a$.
} 

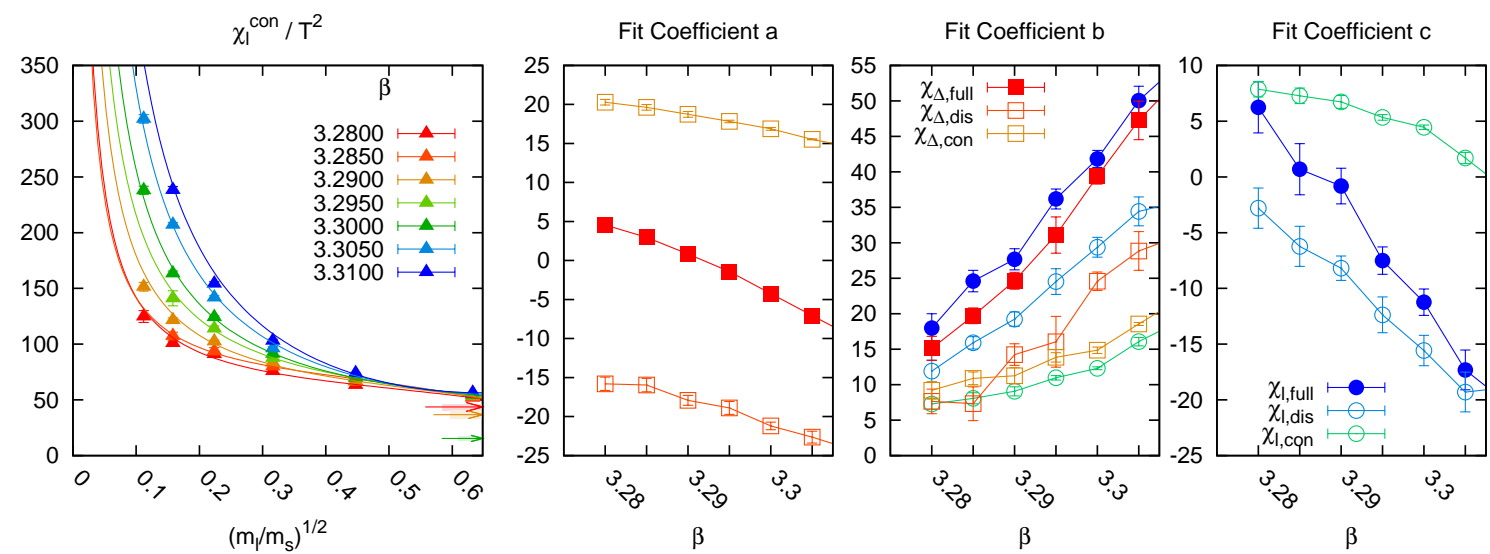

Figure 3: left: Goldstone fit for connected susceptibility, right: fit coefficients $a, b$ and $c$ for the susceptibilities - fit ansatz is chosen in a way that allows direct comparison with fit coefficients of the condensates

can be seen from the magnetic equation of state [5]. For QCD with two light flavors, staggered chiral perturbation theory predicts also Goldstone scaling for the connected susceptibility if taste violations are strong, in contrast to continuum chiral perturbation theory. Our lattice data have confirmed this picture for a coarse lattice, $N_{\tau}=4$. In the future, we will proceed to analyse the effect of taste violations for chiral observables by extending the analysis to $N_{\tau}=8$.

\section{Acknowledgements}

The work has been supported in parts by the U.S. Department of Energy under Contract No. DEAC02-98CH10886 and by the Deutsche Forschungsgemeinschaft under grant GRK 881. I want to thank the members of the RBC-Bielefeld collaboration for helpful discussions.

\section{References}

[1] H. Leutwyler, J. Gasser. Ann. Phys. 158, 1984.

[2] H. Leutwyler, P. Hasenfratz. Nucl. Phys Proc. B 343, 1990.

[3] R. K. P. Zia, D. J. Wallace. Phys. Rev. B 12, 1975.

[4] T. Mendes, J. Engels. Nucl. Phys Proc. Suppl. 83, 2000.

[5] S. Ejiri et al. arXiv:0909.5122, 2009.

[6] P. Gerber, H. Leutwyler. Nuc. Phys. B 321, 1989.

[7] J. Gasser, H. Leutwyler. Nucl. Phys. B 250, 1985.

[8] A. V. Smilga, J. Stern. Phys. Lett. B 318, 1993.

[9] A. V. Smilga, J. J. M. Verbaarschot. Phys. Rev. D 54, 1996.

[10] B. Sturm, U. M. Heller, F. Karsch. Phys. Rev. D 60, 1999.

[11] W. Lee, S. R. Sharpe. Phys. Rev. D 60, 1999.

[12] C. Aubin, C. Bernard Phys. Rev. D 68, 2003.

[13] C. Bernard, C. DeTar, Z. Fu, S. Prelovsek Phys. Rev. D 76, 2007. 